\title{
Depressão e suporte familiar em pacientes renais crônicos: uma revisão narrativa
}

\author{
Depression and family support in chronic renal patients: a narrative review
}

Depresión y apoyo familiar em pacientes renales crónicos: uma revisión narrativa

Gustavo Oliveira de Araujo ${ }^{*}$, José Doriberto Freitas ${ }^{2}$, Raimunda Furtado de Sousa ${ }^{1}$, Jefferson da Silva Rodrigues ${ }^{1}$, Antonia Mavilla Sales da Cunha ${ }^{1}$, Virgínia Maria de Paula Frota Souto ${ }^{1}$, Natalia Lopes Braga².

\section{RESUMO}

Objetivo: Verificar a Depressão e o Suporte Familiar em pacientes renais crônicos. Revisão bibliográfica: Após o levantamento de artigos sobre os temas, Depressão, Doença Renal Crônica (DRC), Hemodiálise e Suporte Familiar, foi verificado que as hemodiálises juntamente com a DRC estão associadas ao surgimento da depressão. Os estudos indicam que isso pode afetar as condições físicas e psicossociais dos pacientes, gerando sobrecarga das comorbidades, maiores complicações da doença, intercorrências hemodialíticas e dependência funcional. Os artigos também mostram que a percepção de um adequado suporte familiar está relacionada ao aumento do senso de segurança em relação à sobrevivência em indivíduos com constantes crises de saúde, podendo reforçar hábitos saudáveis e minimizar os níveis de depressão. Considerações finais: Foi constatado elevado índice de depressão em Pacientes Renais Crônicos (PRC), o que acaba por dificultar o tratamento hemodialítico, sendo este o mais utilizado atualmente. Os pacientes que percebem ou que têm um melhor suporte familiar têm minimizados os seus sintomas depressivos e alcançam melhores resultados durante a terapia substitutiva.

Palavras-chave: Depressão, Doença renal crônica, Suporte familiar, Hemodiálise.

\begin{abstract}
Objective: To verify Depression and Family Support in chronic kidney patients. Bibliographic review: After collecting articles on the themes, Depression, Chronic Kidney Disease (CKD), Hemodialysis and Family Support, it was found that hemodialysis together with CKD are associated to the onset of depression. Studies indicate that this can affect the physical and psychosocial conditions of patients, generating overload of comorbidities, greater complications of the disease, hemodialysis complications and functional dependence. The articles also show that the perception of adequate family support is related to an increased sense of security in relation to survival in individuals with constant health crises, which can reinforce healthy habits and minimize levels of depression. Final considerations: A high rate of depression was found in Chronic Kidney Patients (CRP), which ends up hampering hemodialysis treatment, which is currently the most used. Patients who notice or have better family support have minimized their depressive symptoms and achieve better results during replacement therapy.
\end{abstract}

Keywords: Depression, Chronic kidney disease, Family support, Hemodialysis.

\section{RESUMEN}

Objetivo: Verificar Depresión y Apoyo Familiar en pacientes renales crónicos. Revisión bibliográfica: Tras recopilar artículos sobre los temas Depresión, Enfermedad Renal Crónica (ERC), Hemodiálisis y Apoyo Familiar, se encontró que las hemodiálisis junto con la ERC se asocian al início de depresión. Los estudios indican que esto puede afectar las condiciones físicas y psicosociales de los pacientes, generando sobrecarga de comorbilidades, mayores complicaciones de la enfermedad, complicaciones de hemodiálisis y dependencia funcional. Los artículos también muestran que la percepción de un apoyo familiar adecuado se relaciona con una mayor sensación de seguridad en relación a la supervivencia en individuos con crisis de

${ }^{1}$ Faculdade leducare (FIED), Tianguá - CE. ^E-mail: guguaraujo221@gmail.com

2 Universidade de Fortaleza (UNIFOR), Fortaleza - CE. 
salud constantes, lo que puede reforzar hábitos saludables y minimizar los niveles de depresión. Consideraciones finales: Se encontró una alta tasa de depresión en Pacientes Renales Crónicos (PCR), lo que termina dificultando el tratamiento de hemodiálisis, que es el más utilizado en la actualidad. Los pacientes que notan o tienen un mejor apoyo familiar han minimizado sus síntomas depresivos y logran mejores resultados durante la terapia de reemplazo.

Palabras clave: Depresión, Enfermedad renal crónica, Apoyo familiar, Hemodiálisis.

\section{INTRODUÇÃO}

Atualmente a Doença Renal Crônica (DRC) é considerada um sério problema de saúde pública em todo o mundo, com perspectiva de aumento substancial no número de pacientes tratados com diálise (OTTAVIANI AC, et al., 2016). Caracterizada pela perda permanente da função dos rins é considerada pelos pacientes "um pesadelo" devido às dificuldades enfrentadas no tratamento (COSTA FG e COLTINHO MD, 2016). A DRC tem sido relacionada a vários fatores, como hipertensão arterial e diabetes. Fatores como questões socioeconômicas, raciais e de gênero são também determinantes para essa incidência (MARINHO AWGB et al., 2017). Além disso, $20 \%$ dos pacientes passam a apresentar sintomas depressivos a partir da descoberta da doença (COSTA FG e COLTINHO MD, 2014).

A hemodiálise é a Terapia Renal Substitutiva (TRS) mais utilizada no Brasil. É o tratamento mais comum para os pacientes portadores de DRC, ela consiste na filtração do sangue, onde o mesmo é retirado aos poucos do organismo por meio de uma agulha especial, ou por meio de um cateter localizado numa veia no centro do pescoço, o sangue é bombeado por meio de uma máquina e após isso, passa por um processo de filtração que retira toda a toxina e água em excesso no organismo, em seguida o sangue retorna ao corpo. Apenas clínicas especializadas realizam esse tratamento, que deve ser feito no mínimo 3 vezes por semana com duração de 3-4 horas aproximadamente (HERNANDES F, 2009).

Os Pacientes Renais Crônicos (PRC) quando submetidos à hemodiálise passam a lidar com perdas e mudanças estressantes nas funções de imagem e nas funções orgânicas, e como consequência, podem tornar- se ansiosas e deprimidas (SOUSA L, 2017). Preto CR et al. (2019) apontam que a DRC juntamente com a Hemodiálise está associada ao surgimento da depressão, afetando consequentemente as condições físicas e psicossociais dos pacientes, gerando sobrecarga das comorbidades, maiores complicações da doença, intercorrências hemodialíticas e dependência funcional.

Os estudos sobre depressão têm crescido nos últimos anos e os pesquisadores da saúde mental consideram a depressão o fenômeno clínico que mais acomete a população. Atualmente, mais de 300 milhões de pessoas de faixas etárias diferentes sofrem com esse transtorno que pode gerar uma série de problemas, como perda de energia, mudanças no apetite, aumento ou redução do sono, ansiedade, perda de concentração dentre outros, causando danos pessoais e também para aqueles de seu convívio (ORGANIZAÇÃO PAN- AMERICANA DA SAÚDE (OPAS), 2019).

Em geral, a depressão é frequentemente diagnosticada em virtude de outros transtornos, dentre os quais os da personalidade (ex. Boderline), os relacionados ao uso de substâncias (ex. Alcoolismo) e os de ansiedade (ex. Transtorno de Ansiedade Generalizada). Nos Estados Unidos a prevalência da depressão na população é de $16,6 \%$ ao longo da vida adulta, onde a cada ano 6,7\% recebe um diagnóstico de depressão, sendo $30,4 \%$ desses casos classificados como graves. As mulheres são $70 \%$ mais propensas que os homens a vivenciarem a depressão em algum momento da vida, sendo aos 32 anos a idade média para o início da doença. Estudos realizados em diferentes países indicam a gravidade da doença, seu caráter epidêmico, elevado custo para os serviços de saúde e sua relação com fatores de vulnerabilidade (WHITBOURNE SK e HALGIN RP, 2015).

Segundo Ross CE e Mirowsky J (2002), a percepção de um adequado suporte familiar está relacionada ao aumento do senso de segurança em relação à sobrevivência em indivíduos com constantes crises de saúde, podendo reforçar hábitos saudáveis. Além disso, esses autores apontaram que as pessoas que percebem um suporte emocional por parte da família possuem uma expectativa de vida maior por 
apresentarem esperança de vida mais elevada. Segundo Baptista MN (2005), um alto nível de percepção de suporte familiar está relacionado com baixa prevalência de transtornos ansiosos e de humor, podendo ter influência positiva nos tratamentos.

No que se refere ao suporte familiar, para Marques MT (2016), a definição de família envolve as relações sociais que funcionam como redes de suporte que cercam o indivíduo. Essa definição é conhecida como modelo de comboio, que vincula o conceito de família ao suporte recebido pelo sujeito. O suporte é entendido como o amortecimento do impacto ocasionado por agentes estressores na vida das pessoas. Por essa razão, o suporte familiar tem sido útil nos estudos de resiliência psicológica, pois o suporte das relações humanas é importante para a terapêutica no âmbito da saúde.

Percebe-se que desde o momento do diagnóstico, o paciente renal se depara com uma série de questões que põem em evidência sua problemática pessoal e familiar, muitos acabam por perder seus empregos e, também, laços com seus grupos sociais. Nesse sentido, o suporte social pode ser uma ferramenta importante, por sua comprovada relação com o desenvolvimento e a manutenção de áreas psicológicas do ser humano. É um constructo multidimensional que envolve a relação entre o meio ambiente e o indivíduo, e seus efeitos no comportamento humano, sendo a família considerada o suporte social mais relevante para o ser humano (BAPTISTA MN, 2005). Diante do exposto, o objetivo do estudo é verificar a Depressão e o Suporte Familiar em pacientes renais crônicos.

\section{REVISÃO BIBLIOGRÁFICA}

Os estudos apontam diversos fatores que podem ocasionar o surgimento da depressão como, por exemplo, desemprego e isolamento e causas biológicas (PELUSO ETP e BLAY SL, 2008). De acordo com Tavares AP, et al. (2020), a intensidade de sintomas dos pacientes com DRC avançada, em diálise, são muito semelhantes aos de outras doenças crônicas, os pacientes também têm uma sobrevida não superior àquela de muitas doenças malignas, o que gera grande impacto na Qualidade de Vida Relacionada à Saúde (QVRS), e na expectativa de vida, e alto custo pessoal e social dos pacientes.

Esses pacientes apresentam sintomas depressivos, sendo eles fatores de risco para a diálise e doença cardiovascular, aumento da morbidade, ou redução da qualidade de vida, tornando as chances de suicídio dez vezes maiores do que em relação à população geral. No que se refere ao aparecimento de sintomas psicopatológicos, é de essencial importância à atenção dos profissionais em relação a esse público, para que haja um diagnóstico precoce evitando assim maiores complicações (JUNIOR EV et al., 2018).

Preto CR, et al. (2019) realizaram um estudo que visou verificar a associação entre variáveis sociodemográficas, clínicas, hábitos de vida e capacidade funcional com indicativos de depressão de PRC em hemodiálise. O mesmo pôde comprovar que $60,3 \%$ dos pacientes apresentaram sintomas depressivos e que houve associação entre indicativos de depressão com sexo feminino, maior número de comorbidades e intercorrências pós-hemodiálise, sintomas físicos, emocionais e comportamentais como deixar de realizar atividades habituais e a necessidade de auxílio no dia a dia.

Muscat $P$, et al. (2020) delinearam entre 2015 e 2019 uma pesquisa para avaliar se as percepções da doença impactam a mortalidade em pacientes com DRC incidente pré-diálise, participaram 200 pacientes das clínicas ambulatoriais de Nefrologia do Hospital Mater Dei, Malta. Os resultados obtidos evidenciam que as crenças sobre a doença aumentam o risco de mortalidade em pacientes com DRC, independentemente de outras variáveis clínicas e demográficas. Assim, também o problema pode estar associado ao sofrimento, pois ao perceber a amplitude da gravidade e interpretar os sintomas têm-se níveis mais elevados de angústia entre os pacientes com DRC, com isso tornam-se preditores de comportamentos que podem desencadear a depender da frequência, uma instabilidade ou maior intensidade de quadros psicopatológicos como a depressão.

Agrawaal KK, et al. (2019) concluíram um estudo com 100 pacientes em hemodiálise, que tinha o objetivo de verificar a prevalência de depressão nestes, para tal foi utilizado o questionário de saúde do paciente-9, que constatou a prevalência de depressão em $78 \%$ dos pacientes, sendo a fadiga o sintoma mais comum, presente em $82 \%$ dos pacientes. 
Em um estudo realizado por Murilo-Zamora E, et al. (2016), em um hospital urbano do Instituto Mexicano de Previdência Social, teve como objetivo verificar a prevalência de depressão em pacientes mexicanos com DRC em estágio terminal e em hemodiálise de manutenção, utilizando o Inventário de Depressão de Beck (IDB), foi aplicado em 81 pacientes, entre 30 e 69 anos, onde constatou-se alta prevalência de depressão entre pacientes adultos acometidos com esta patologia.

Bastos DS, et al. (2016) utilizaram um protocolo com dados sociodemográficos, o IDB e o Inventário de Percepção Suporte Familiar (IPSF) com o objetivo de investigar a sintomatologia depressiva e a percepção do suporte familiar em idosos e adultos com DRC. O IDB apontou níveis de depressão mínima e o IPSF, níveis elevados de percepção de suporte familiar, não sendo encontradas diferenças significativas entre os grupos. Houve uma correlação negativa $(r=-0,36 ; p=0,01)$ entre o BDI-II e o IPSF, indicando que quanto maior a percepção de suporte familiar, menor a sintomatologia depressiva.

Starczewska M, et al. (2020) executaram uma pesquisa em Koszalin, cidade da Polônia, onde objetivouse avaliar o aumento do estresse e a ocorrência de transtornos depressivos em pacientes hemodialisados utilizando como métodos a Escala de Estresse (PSS-10) e o IDB. A amostra contou com a participação de 102 pacientes, onde com a análise dos resultados, pode-se concluir que a maioria dos pacientes tratados com hemodiálise é acometida por depressão leve e altos níveis de ansiedade.

Brito DCS, et al. (2019) usaram o IDB, Questionário de Saúde Curto de 36 itens (SF-36), Índice de Comorbidade de Charlson e Avaliação Subjetiva Global para analisarem os níveis de depressão e ansiedade de 205 pacientes, na qual 130 deles estavam em diálise e 75 deles haviam sido submetidos a transplante. concluiu-se que a prevalência de sintomas de depressão fora de $31,2 \%$ na amostra geral estudada. Da mesma forma, depreendeu-se que a depressão afetou quase três vezes mais pacientes em diálise do que pacientes transplantados, pois a saúde mental dos pacientes em hemodiálise e diálise peritoneal é de certa forma mais impactada do que a dos pacientes transplantados, por conta das imposições à rotina rígida do tratamento de diálise, somado às limitações às atividades sociais e familiares.

Por sua vez, os pacientes que já realizaram o transplante renal possuem uma maior sensação de bemestar visto a liberdade de diálise e restrições, o que tem impacto positivo na autopercepção da saúde. Ainda, ao considerar um estado nutricional ruim e estado geral de saúde constatou-se o agravamento da depressão, considerando que o desenvolvimento de transtornos mentais é multideterminado seja pela situação social do paciente, seu quadro clínico, limitação incapacidade de trabalho, condições financeiras ruins e aumento do isolamento (BRITO DCS, et al., 2019).

Cwiek A, et al. (2017), através de um estudo que tinha por objetivo investigar e comparar a prevalência de depressão entre pacientes hemodialisados e não hemodialisados, obteve como resultado a alta prevalência de sintomas de ansiedade e depressão em pacientes com DRC, sendo em sua maioria em pacientes hemodialisados. Segundo os pesquisadores, esses resultados indicam a importância do monitoramento do estado mental dos pacientes com DRC, além do fornecimento de suporte psicológico.

Com o objetivo de determinar a prevalência de sintomas depressivos e explorar fatores relacionados à depressão em pacientes em tratamento de hemodiálise no norte da China, Liu X, et al. (2017) utilizaram como método, o desenho de pesquisa transversal, com uma amostra de 227 pacientes, entre dezembro de $2012 \mathrm{e}$ março de 2013. Ao desfecho do estudo pode-se comprovar que a prevalência de sintomas depressivos é alta em pacientes com DRC em hemodiálise. Constatou-se também que atividades da vida diária, apoio familiar, estilo de enfrentamento "aceitação-resignação" e resiliência do ego foram independentes associados com sintomas depressivos.

Souza FTZ e Oliveira JHA (2017) fizeram uma pesquisa que avaliou sintomas de depressão e ansiedade em 61 pacientes renais crônicos em tratamento conservador, os resultados constataram associações existentes entre as variáveis: depressão, ansiedade e estágios da DRC, sendo perceptíveis as diferenças obtidas em pacientes que se encontravam em estágio inicial e final da DRC, com maior probabilidade de desenvolvimento de transtornos de humor e ansiosos. 
Uma pesquisa realizada por Stasiak CES, et al. (2014) com objetivo de comparar a prevalência de ansiedade e depressão em pacientes que realizam hemodiálise e Diálise Peritoneal (DP), levando em conta comorbidades que possam contribuir para isso. Pôde constatar utilizando a Escala Hospitalar de Ansiedade e Depressão (EHAD), Inventário de Ansiedade de Beck (BAI), e o IDB a prevalência de depressão em 22,6\% dos pacientes no BDI e em 9,3\% na EHAD, e ansiedade em 25,7\% no BAI e em 11,7\% na EHAD. No grupo em DP, 29,6\% dos pacientes apresentaram depressão no BDI e 14,8\% na EHAD, e ansiedade em 11,1\% no BAl e em nenhum na EHAD.

Silva SM, et al. (2016) finalizaram um estudo que teve como objetivo avaliar o suporte social instrumental e emocional de PRC em tratamento hemodialítico que apontou bom suporte recebido. Esses resultados se justificam devido os pacientes com DRC apresentarem elevado apoio social, tanto instrumental quanto emocional, sendo a principal forma de apoio proveniente da família. Entre as fontes mais frequentes de suporte social instrumental e emocional mencionadas pelos participantes encontram-se os parceiros, cônjuge, companheiro ou namorado e amigos.

Uma pesquisa elaborada por Simms RJ, et al. (2016), cujo o objetivo era avaliar a qualidade de vida, humor, apoio social percebido e risco psicossocial de ter um diagnóstico de Doença Renal Policística Dominante (DRPD) em pacientes de um importante centro de nefrologia. Apontou como resultado uma qualidade de vida significativamente pior aumentando o risco psicossocial com marcadores de progressão da doença em pacientes com DRPD sendo relatada a presença significativa de depressão em $22 \%$ dos pacientes.

Em outro estudo planejado por Silva RAR, et al. (2016), que objetivou conhecer as estratégias de enfrentamento utilizadas por PRC submetidos ao tratamento hemodialítico, frente às dificuldades inerentes à doença, constatou que a família gerou o suporte psicoemocional necessário para superar os momentos de tensão e desânimo que surgiram durante a caminhada. No estudo, o apoio familiar configurou-se como importante ferramenta de adaptação psicológica aos eventos estressores decorrentes da terapêutica hemodialítica.

Madeiro AC, et al. (2010) executaram um estudo que se propôs a avaliar a adesão do paciente com Insuficiência Renal Crônica (IRC), ao tratamento de hemodiálise. O autor constatou que o fato do paciente residir com a família ou ter alguns companheiros pode ajudar a melhorar o apoio social relacionado a complicações devido ao IRC. Mostrou também ser essencial a participação da família, pois é necessário funções de proteção e socialização dos seus membros, já que a família, como unidade, desenvolve um sistema de valores, crenças e atitudes com saúde e doença que são expressas e demonstradas através do comportamento e saúde de seus membros.

A partir de um estudo delineado por Andrade SV, et al. (2015), com objetivo averiguar se existe diferença nos sintomas de desesperança, ideação suicida e depressão entre PRC em hemodiálise ou transplantados e se variáveis sociodemográficas como atividade laboral, ter dependentes, sexo e estado civil interferem nos sintomas. Foi possível constatar que não houve diferença significativa na intensidade dos sintomas de desesperança, ideação suicida e depressão entre pacientes estáveis em hemodiálise e transplantados, porém não exercer atividade laboral e receber transplante de doador falecido levou a mais sintomas depressivos e que a prevalência de ideação suicida e sintomas depressivos, nas duas modalidades, merece atenção e indica a necessidade de monitorização e cuidados nesses pacientes.

Em outro estudo planejado por Carvalho AR e Barbosa MR (2016), que pretendia investigar e compreender a prevalência da depressão em doentes hemodialisados e qual a sua relação com a imagem corporal e a sexualidade. Os autores indicaram haver um impacto negativo da hemodiálise na depressão, insatisfação corporal e na satisfação com a sexualidade, sendo estes resultados um alerta para a importância da intervenção psicológica na população de pacientes com IRC submetidos à hemodiálise, sobretudo na insatisfação corporal e na depressão.

Santos TRS, et al. (2021) concluíram uma pesquisa que objetivou analisar o perfil de saúde relacionada à depressão e qualidade de sono de pacientes com DRC em hemodiálise. Na avaliação da escala hospitalar 
de ansiedade e depressão 8,5\% dos pacientes foram identificados com "depressão", os quais tinham maior proporção de diabetes e hipertensão em relação aos demais com diferença considerável. O grupo que declarou sentir o impacto da doença apresentou mais sonolência e maior probabilidade para ansiedade e depressão com diferença significativa em relação ao grupo que declarou não sentir impacto com a doença.

Estudo planejado por Costa FG e Coutinho MPL (2016) que pretendia mensurar a sintomatologia depressiva em PRC e seus cuidadores/familiares e relacioná-la às variáveis biossociodemográficas, foi possível comprovar um índice de sintomas depressivos de $20 \%$ nos pacientes, com o principal índice entre pacientes solteiros (50\%), em parentes, a prevalência de sintomas depressivos foi de $46 \%$, observando correlação positiva entre o tempo de cuidado e a depressão.

Uma pesquisa elaborada por Fernandes D, et al. (2020), cujo objetivo era avaliar a qualidade de vida de pacientes portadores de DRC em TRS, modalidade de hemodiálise, identificando os componentes e seus respectivos domínios comprometidos. Pode-se concluir que a DRC causa impacto nos diferentes domínios dos componentes físico e mental nos pacientes em tratamento hemodialítico e tais comprometimentos podem implicar em significativo impacto, desempenho e percepção destes indivíduos sobre sua qualidade de vida frente à doença e seu tratamento.

Pereira E, et al. (2021) fizeram um estudo com intuito de descrever o percurso de estrangeiros DRC em busca de tratamento no Sistema Único de Saúde (SUS), em um município fronteiriço. O mesmo resultou em sete categorias temáticas que indicaram: pouca percepção da doença; suporte familiar dificultado ao longo do tratamento; aspectos sociais que envolvem trabalho, renda e moradia como fonte de angústia; procura por tratamentos alternativos; busca por assistência à saúde no exterior interrompida por questões financeiras; caminho percorrido à procura de assistência à saúde no Brasil facilitado por familiares e redes sociais, sendo o entendimento referente à doença um preditivo na prática do autocuidado referente a vários aspectos dentre eles, a manifestação da depressão.

Cremasco GS e Baptista MN (2018) realizaram um estudo que teve como objetivo analisar produções científicas nacionais e internacionais a respeito da depressão e da DRC entre os anos de 2006 e 2016 a partir de uma revisão integrativa da literatura. O estudo verificou que a prevalência de sintomatologia depressiva em PRC variou de $7,8 \%$ a $83,49 \%$.

Pesquisa realizada por Santos ACM e Nakasu MVP (2017) que visou investigar a prevalência de sintomas de estresse e depressão em PRC sob tratamento dialítico no Hospital Escola de Itajubá, no estado de Minas Gerais, Brasil, constatou que a presença de sintomas de estresse e depressão na amostra foi de $32 \%$ e $34 \%$, onde sinais e sintomas indicadores de estresse nesses pacientes se revelaram comuns, evidenciando a vulnerabilidade dos mesmos a uma maior prevalência de depressão.

O estudo objetivou verificar, por meio de uma revisão de literatura, a depressão e suporte familiar em Doentes Renais Crônicos (DRC). Tratou-se de uma revisão narrativa da literatura, envolvendo o período de 2010 a 2021. Foram identificados no período analisado, 27 trabalhos. Desses, foram escolhidos 26 que especificamente estavam relacionados com impactos na saúde mental, especialmente a depressão e a importância do suporte familiar em DRC. Os estudos apontaram uma alta prevalência da depressão nesta população e que um adequado suporte familiar é fundamental para minimizar os impactos da doença.

\section{CONSIDERAÇÕES FINAIS}

Os resultados da pesquisa apontam para um alto índice de depressão em pacientes com doença renal. Foram observados também alguns aspectos decorrentes da realidade da DRC e as etapas que o doente percorre a partir do seu diagnóstico. É possível concluir que os pacientes que percebem ou que têm um melhor suporte familiar têm minimizados os seus sintomas depressivos e alcançado melhores resultados durante a terapia substitutiva. $O$ estudo mostra que é necessário focar em estratégias, planejamentos e intervenções que tragam a melhoria da saúde e qualidade de vida dessas pessoas. Pode-se pensar também na necessidade de uma atuação governamental que trabalhe na perspectiva da promoção da saúde, bem como o engajamento de familiares no cuidado a estes pacientes. 


\section{REFERÊNCIAS}

1. AGRAWAAL KK, et al. Prevalência de depressão em pacientes com DRC 5 em hemodiálise em um centro de atenção terciária no nepal, Revista da Associação Médica do Nepal, 2019; 57(21): 172-175.

2. ANDRADE SV, et al. Desesperança, ideação suicida e depressão em pacientes renais crônicos em tratamento por hemodiálise ou transplante. J Bras Nefrol, 2015; 37(1): 55-63.

3. BAPTISTA MN. Desenvolvimento do Inventário de Percepção de Suporte Familiar (IPSF): Estudos psicométricos preliminares. Psico-USF, 2005; 10(1): 11-19.

4. BASTOS DS, et al. Sintomas depressivos e suporte familiar em idosos e adultos em hemodiálise. Revista Psicologia: Teoria e Prática, 2016; 18(2): 103-116.

5. BRITO DCS, et al. Depression and anxiety among patients undergoing dialysis and kidney transplantation: a cross-sectional study. São Paulo Med. J, 2019; 137(2): 137-147.

6. CARVALHO AR, BARBOSA MR. A depressão nos doentes hemodialisados: o papel da satisfação corporal da sexualidade. Revista portuguesa de Saúde Pública, 2016; 34(2): 144-153.

7. COSTA FG, COUTINHO MP. Doença renal crônica e depressão: um estudo psicossociológico com pacientes em hemodiálise. Psicologia e Saber Social, 2016; 5(1): 78-87.

8. COSTA FG, COUTINHO MP. Hemodiálise e depressão: representação social dos pacientes. Psicol. estud, 2014; 19(4): 657667.

9. COSTA FG, COUTINHO MP. Síndrome depressiva: um estudo com pacientes e familiares no contexto da doença renal crônica. Estudos Interdisciplinares em Psicologia, 2016; 7(1): 38-55.

10. CREMASCO GS, BAPTISTA MN. Depressão e doença renal crônica: revisão integrativa da literatura. Revista Psicologia: Teoria e Prática, 2018; 20(3): 343-359.

11. CWIEK A, et al. Association between depression and hemodialysis in patients with chronic kidney disease. Psychiatria Danubina, 2017; 29(3): 499-503.

12. FERNANDES D, et al. Qualidade de vida de pacientes em terapia renal substitutiva: uma análise da doença renal crônica e perfil populacional de risco. Revista Eletrônica Acervo Saúde, 2020; 12(12): 1-9.

13. HERNANDES F, et al. Mini dicionário do paciente renal: tratamento conservador. Abbott Center, 2011; 1-15.

14. JÚNIOR EV, et al. Impacto de los factores asociados a los síntomas depresivos sobre la salud en ancianos en hemodiálisis. Enfermería Actual en Costa Rica, 2018; 6(35): 1-11.

15. LIU X, et al. Prevalence and related factors of depressive symptoms in hemodialysis patients in northern China. BMC psychiatry, 2017; 17(1): 1-7.

16. MADEIRO AC, et al. Adesão de portadores de insuficiência renal crônica ao tratamento de hemodiálise. Acta Paulista de Enfermagem, 2010; 23(4): 547-548.

17. MARINHO AWGB, et al. Prevalência de doença renal crônica em adultos no Brasil: revisão sistemática da literatura. Cad. Saúde Coletiva, 2017; 25(3): 379-388.

18. MARQUES MT. Suporte familiar e uso de drogas na perspectiva do indivíduo em tratamento: Um caminho para o cuidado. (Pós-graduação em neuropsiquiatria e ciências do comportamento) - Departamento de Neuropsiquiatria e Ciências do Comportamento da Universidade Federal de Pernambuco, Pernambuco, 2016; 88p.

19. MURILLO-ZAMORA E, et al. Prevalência de depresión entre pacientes con enfermedad terminal en hemodiálisis de mantenimiento. Revista Médica del Instituto Mexicano del Seguro Social, 2016; 4(4): 429-433.

20. MUSCAT P, et al. Illness perceptions predict mortality in patients with predialysis chronic kidney disease: a prospective observational study. BMC Nephrol, 2020; 21(53̧7): 1-8.

21. ORGANIZAÇÃO PAN-AMERICANA DA SAÚDE (OPAS). 2019. In: Folha Informativa - Depressão. Disponível em: https://www.paho.org/bra/index.php?option=com_content\&view=article\&id=5635:folha-informativa-depressao\&ltemid=1095. Acesso em: 19 out. 2020.

22. OTTAVIANI AC, et al. Associação entre ansiedade e depressão e a qualidade de vida de pacientes renais crônicos em hemodiálise. Texto Contexto Enfermagem, 2016; 25(3): 1-8.

23. PELUSO ETP, BLAY SL. Percepção da depressão pela população da cidade de São Paulo. Revista Saúde Pública, 2008; 42(1): 8-41.

24. PEREIRA E, et al. Percurso de estrangeiros doentes renais crônicos na busca por assistência à saúde em área fronteiriça. Revista Brasileira de Enfermagem, 2021; 74(01): 1-8.

25. PRETTO CR, et al. Depressão e pacientes renais crônicos em hemodiálise: fatores associados. Revista Brasileira de Enfermagem, 2019; 73(1): 1-8.

26. ROSS CE, MIROWSKY J. Family relationships, social support, and subjective life expectancy. Journal of Health and Social Behavior, 2002; 43(4): 469-89.

27. SANTOS ACM, NAKASU MVP. Prevalência de sintomas de estresse e depressão em pacientes renais crônicos submetidos à hemodiálise em um hospital escola do sul de Minas Gerais. Revista Ciências em Saúde, 2017; 7(2): 17-22.

28. SANTOS TSRD, et al. Sono e depressão em pacientes com doença renal crônica em hemodiálise. Anais do Seminário Científico do Programa de Pós-Graduação em Promoção da Saúde UNISC, 2021.

29. SILVA RAR, et al. Estratégias de enfrentamento utilizadas por pacientes renais crônicos em tratamento hemodialítico. Escola Anna Nery, 2016; 20(1): 147-154.

30. SILVA SM, et al. Suporte social de adultos e idosos renais crônicos em hemodiálise. Revista Latino- Americana de Enfermagem, 2016; 24(2752): 1-6.

31. SIMMS RJ, et al. Increased psychosocial risk, depression and reduced quality of life living with autosomal dominant polycystic kidney disease. Nephrol Dial Transplant, 2016; 31:1140-1144.

32. SOUSA L, et al. A depression anxiety stress scale em pessoas com Doença renal crónica. Revista Portuguesa de Enfermagem de Saúde Mental, 2017; 17: 50-57.

33. SOUZA FTZ, OLIVEIRA JHA. Sintomas Depressivos e Ansiosos no Paciente Renal Crônico em Tratamento Conservador. Revista Psicologia e Saúde, 2017; 09(3): 17-31.

34. STARCZEWSKA M, et al. Assessment of stress level and prevalence of depression among haemodialyzed patients. Family Medicine \& Primary Care Review, 2020; 22(3): 216-221.

35. STASIAK CES, et al. Prevalência de ansiedade e depressão e suas comorbidades em pacientes com doença renal crônica em hemodiálise e diálise peritoneal. J. Bras. Nefrol, 2014; 36(3): 325-331.

36. WHITBOURNE SK, HALGIN RP. Psicopatologia: Perspectivas clínicas dos transtornos psicológicos. 7ª Ed. Porto Alegre: AMGH, 2015; 458p. 